\title{
On the existence of Feller semigroups with discontinuous coefficients
}

\author{
Kazuaki Taira* \\ Institute of Mathematics, University of Tsukuba, Tsukuba 305-8571, Japan \\ E-mail: taira@math.tsukuba.ac.jp \\ Dedicated to Professor Koichi Uchiyama on the occasion of his 60th birthday
}

\begin{abstract}
This paper is devoted to the functional analytic approach to the problem of existence of Markov processes in probability theory. More precisely, we construct Feller semigroups with Dirichlet conditions for second-order, uniformly elliptic integro-differential operators with discontinuous coefficients. Rephrased, we prove that there exists a Feller semigroup corresponding to such a diffusion phenomenon that a Markovian particle moves both by jumps and continuously in the state space until it dies at the time when it reaches the boundary.
\end{abstract}

\section{Introduction}

Let $\Omega$ be a bounded domain in Euclidean space $\mathbf{R}^{N}, N \geq 3$, with boundary $\partial \Omega$ of class $C^{1,1}$. In this paper we assume that the domain $\Omega$ is convex.

Now we consider a second-order, elliptic integro-differential operator $W$ with real discontinuous coefficients of the form

$$
\begin{aligned}
W u(x)= & A u(x)+S u(x) \\
:= & \left(\sum_{i, j=1}^{N} a^{i j}(x) \frac{\partial^{2} u}{\partial x_{i} \partial x_{j}}(x)+\sum_{i=1}^{N} b^{i}(x) \frac{\partial u}{\partial x_{i}}(x)+c(x) u(x)\right) \\
& +\int_{\bar{\Omega}} s(x, d y)\left[u(y)-u(x)-\sum_{j=1}^{N}\left(y_{j}-x_{j}\right) \frac{\partial u}{\partial x_{j}}(x)\right] .
\end{aligned}
$$

Here:

(1) $a^{i j}(x) \in \mathrm{VMO} \cap L^{\infty}\left(\mathbf{R}^{N}\right), a^{i j}(x)=a^{j i}(x)$ almost all $x \in \Omega$ and there exists a constant $\lambda>0$ such that

$$
\lambda^{-1}|\xi|^{2} \leq \sum_{i, j=1}^{N} a^{i j}(x) \xi_{i} \xi_{j} \leq \lambda|\xi|^{2} \text { for almost all } x \in \Omega \text { and all } \xi \in \mathbf{R}^{N} .
$$

2000 Mathematics Subject Classification. Primary 47D07, 35J25; Secondary 47D05, 60J35, 60J60

Keyword. Feller semigroup, Dirichlet condition, VMO function

* Supported in part by Grant-in-Aid for General Scientific Research (No. 16340031), Ministry of Education, Culture, Sports, Science and Technology, Japan. 
(2) $b^{i}(x) \in L^{\infty}(\Omega)$.

(3) $c(x) \in L^{\infty}(\Omega)$ and $c(x) \leq 0$ for almost all $x \in \Omega$.

(4) $\{s(x, d y)\}_{x \in \Omega}$ is a family of non-negative, Borel measures on the closure $\bar{\Omega}=\Omega \cup \partial \Omega$ which satisfy the following four conditions:

(4-a) For each Borel subset $E$ of $\bar{\Omega}$, the function $x \mapsto s(x, E)$ is Borel measurable on $\Omega$.

(4-b) $s(x,\{x\})=0$ for all $x \in \Omega$.

(4-c) For every small $\varepsilon>0$, there exists a bounded function $\omega(\varepsilon)$ such that

$$
\sup _{x \in \Omega} \int_{\{y \in \bar{\Omega}:|y-x| \leq \varepsilon\}} s(x, d y)|y-x|^{2} \leq \omega(\varepsilon)
$$

and that

$$
\lim _{\varepsilon \downarrow 0} \omega(\varepsilon)=0
$$

(4-d) For every small $\varepsilon>0$, the quantity

$$
C_{\varepsilon}=\sup _{x \in \Omega} \int_{\{y \in \bar{\Omega}:|y-x|>\varepsilon\}} s(x, d y)|y-x|
$$

is finite.

The integro-differential operator $W$ is called a second-order Waldenfels operator (see [1, Chapter 2]). The differential operator $A$ is called a diffusion operator which describes analytically a strong Markov process with continuous paths in the interior $\Omega$ such as Brownian motion. The integral operator $S$ is called a second-order Lévy operator which is supposed to correspond to the jump phenomenon in the closure $\bar{\Omega}$. In this context, conditions (1.2), (1.3) and (1.4) imply that any Markovian particle does not move by jumps so far.

Remark 1.1. Since the domain $\Omega$ is convex, the Lévy operator $S$ may be globally defined by using Taylor's formula as in formula (1.1).

The first purpose of this paper is to prove an existence and uniqueness theorem for the following non-homogeneous Dirichlet problem in the framework of Sobolev spaces of $L^{p}$ style:

$$
\begin{cases}W u=f & \text { in } \Omega \\ \gamma_{0} u=\varphi & \text { on } \partial \Omega .\end{cases}
$$

The next theorem is a generalization of Bony [2, Corollaire 2] to the $V M O$ case:

Theorem 1.1. Let $N<p<\infty$. Then the mapping

$$
\mathcal{W}=\left(W, \gamma_{0}\right): W^{2, p}(\Omega) \longrightarrow L^{p}(\Omega) \bigoplus B^{2-1 / p, p}(\partial \Omega)
$$

is an algebraic and topological isomorphism. In particular, for any $f \in L^{p}(\Omega)$ and any $\varphi \in B^{2-1 / p, p}(\partial \Omega)$, there exists a unique solution $u \in W^{2, p}(\Omega)$ of problem (1.5). 
Remark 1.2. If the leading coefficients $a^{i j}(x)$ are continuous on $\bar{\Omega}$, then results similar to Theorem 1.1 are obtained by Gimbert-Lions [3, Theorem III.1] and Garroni-Menaldi [4, Theorem 3.1.22].

The second purpose of this paper is devoted to the functional analytic approach to the problem of existence of Markov processes in probability theory. More precisely, we construct Feller semigroups with Dirichlet conditions for second-order, uniformly elliptic integro-differential operators with VMO coefficients. Rephrased, we prove that there exists a Feller semigroup corresponding to such a diffusion phenomenon that a Markovian particle moves both by jumps and continuously in the state space until it dies at the time when it reaches the boundary $\partial \Omega$ where the particle is definitely absorbed.

Let $C(\bar{\Omega})$ be the Banach space of all real-valued, continuous functions on the closure $\bar{\Omega}=\Omega \cup \partial \Omega$, equipped with the maximum norm

$$
\|f\|:=\max _{x \in \bar{\Omega}}|f(x)|, \quad f \in C(\bar{\Omega}) .
$$

We introduce a subspace of $C(\bar{\Omega})$, which is associated with Dirichlet condition, by the formula

$$
C_{0}(\bar{\Omega})=\{u \in C(\bar{\Omega}): u=0 \text { on } \partial \Omega\} .
$$

A strongly continuous semigroup $T_{t}$ on the space $C_{0}(\bar{\Omega})$ is called a Feller semigroup if it is non-negative and contractive on $C_{0}(\bar{\Omega})$, that is,

$$
f \in C_{0}(\bar{\Omega}), 0 \leq f(x) \leq 1 \quad \text { on } \bar{\Omega} \Longrightarrow 0 \leq T_{t} f(x) \leq 1 \quad \text { on } \bar{\Omega} .
$$

It is known (see $[5,6]$ ) that if $T_{t}$ is a Feller semigroup on $C_{0}(\bar{\Omega})$, then there exists a unique Markov transition function $p_{t}(x, \cdot)$ on $\Omega$ such that

$$
T_{t} f(x)=\int_{\Omega} p_{t}(x, d y) f(y), \quad f \in C_{0}(\bar{\Omega}) .
$$

Furthermore, it can be shown that the function $p_{t}(x, \cdot)$ is the transition function of some strong Markov process; hence the value $p_{t}(x, E)$ expresses the transition probability that a Markovian particle starting at position $x$ will be found in the set $E$ at time $t$.

The next theorem is a generalization of Bony [2, Théorème 4] to the $V M O$ case:

Theorem 1.2. If $N<p<\infty$, we define a linear operator $\mathfrak{W}$ from $C_{0}(\bar{\Omega})$ into itself as follows:

(1) The domain $D(\mathfrak{W})$ is the space

$$
D(\mathfrak{W})=\left\{u \in W^{2, p}(\Omega) \cap C_{0}(\bar{\Omega}): W u \in C_{0}(\bar{\Omega})\right\} .
$$

(2) $\mathfrak{W} u=W u=A u+S u, u \in D(\mathfrak{W})$.

Here $W u$ is taken in the sense of distributions.

Then the operator $\mathfrak{W}$ is the infinitesimal generator of a Feller semigroup $\left\{T_{t}\right\}_{t \geq 0}$ on $C_{0}(\bar{\Omega})$.

Remark 1.3. The domain $D(\mathfrak{W})$ does not depend on $p$, for $N<p<\infty$ (see Subsection 6.3). 
Remark 1.4. If the leading coefficients $a^{i j}(x)$ are continuous on $\bar{\Omega}$, then results similar to Theorem 1.2 are obtained by Komatsu [7, Theorem 5.2], Stroock [8, Theorem 2.2] and Garroni-Menaldi [9, Chapter VIII, Theorem 3.3]).

The rest of this paper is organized as follows. In Section 2 we recall some basic definitions and results concerning BMO and VMO functions on $\mathbf{R}^{N}$. Section 3 provides a brief description of the basic definitions and results about Feller semigroups and a version of the Hille-Yosida theorem adapted to the present context (Theorem 3.1), which forms a functional analytic background for the proof of Theorem 1.2. In Section 4 we consider the Dirichlet problem for the diffusion operator $A$ in the framework of Sobolev spaces of $L^{p}$ style, and prove an existence and uniqueness theorem for the Dirichlet problem with VMO coefficients (Theorem 4.1). The uniqueness result in Theorem 4.1 follows from a variant of the Bakel'man-Aleksandrov maximum principle in the framework of Sobolev spaces due to Bony [10] (Theorem 4.4). In Section 5 we consider the Dirichlet problem for the Waldenfels operator $W=A+S$ in the framework of Sobolev spaces of $L^{p}$ style, and prove Theorem 1.1. The essential point in the proof is how to estimate the Lévy integral operator $S$ in terms of Sobolev norms (Lemma 5.2). We show that the operator $\mathcal{W}=\left(W, \gamma_{0}\right)$ may be considered as a perturbation of a compact operator to the operator $\mathcal{A}=\left(A, \gamma_{0}\right)$ in the framework of Sobolev spaces. Therefore, the proof of Theorem 1.1 is reduced to the differential operator case which is studied in Section 4. It should be emphasized that the uniqueness result in Theorem 1.1 follows from a variant of the Bakel'man-Aleksandrov maximum principle for the Waldenfels operator $W$ due to Bony [2] (Theorem 5.3). The final Section 6 is devoted to the proof of Theorem 1.2. We make use of an $L^{\infty}$ estimate for the Green operator $G_{\alpha}^{0}$ of the Dirichlet problem to verify all the conditions in Theorem 3.1 for the operator $\mathfrak{W}$ (see estimate (6.10)).

\section{$2 \quad$ BMO and VMO functions}

In this section we recall some basic definitions and results concerning BMO and VMO functions on $\mathbf{R}^{N}$. For more thorough treatments of this subject, the reader might be referred to Garnett [11] and Torchinsky [12].

A function $f \in L_{\text {loc }}^{1}\left(\mathbf{R}^{N}\right)$ is said to be of bounded mean oscillation, $f \in$ BMO, if it satisfies the condition (see [13])

$$
\|f\|_{*}:=\sup _{B} \frac{1}{|B|} \int_{B}\left|f(x)-f_{B}\right| d x<\infty,
$$

where the supremum is taken over all balls $B$ in $\mathbf{R}^{N}$ and $f_{B}$ is the average of $f$ over $B$

$$
f_{B}:=\frac{1}{|B|} \int_{B} f(x) d x .
$$

It should be noticed that the quantity $\|f\|_{*}$ defines a norm on the quotient space $\mathrm{BMO} / \mathbf{R}$. For example, we have

$$
L^{\infty}\left(\mathbf{R}^{N}\right) \subset \mathrm{BMO}
$$

Next we introduce a subspace of BMO functions whose BMO norm over a ball vanishes as the radius of the ball tends to zero. More precisely, if $f \in \mathrm{BMO}$ 
and $r>0$, then we let

$$
\eta(r):=\sup _{\rho \leq r} \frac{1}{|B|} \int_{B}\left|f(x)-f_{B}\right| d x,
$$

where the supremum is taken over all balls $B$ with radius $\rho \leq r$. A function $f \in \mathrm{BMO}$ has vanishing mean oscillation, $f \in \mathrm{VMO}$, if it satisfies the condition (see [14])

$$
\lim _{r \downarrow 0} \eta(r)=0 .
$$

The function $\eta(r)$ will be referred as the VMO modulus of $f$. The assumption that $f \in \mathrm{VMO}$ means a kind of continuity in the average sense, not in the pointwise sense. This property guarantees that VMO functions may be approximated by smooth functions. The next proposition collects some important results concerning VMO functions:

Proposition 2.1. (i) If $f \in \mathrm{VMO}$ with VMO modulus $\eta(r)$, then the usual Friedrichs mollifiers $f_{h}$ converge to $f$ in the BMO norm as $h \downarrow 0$, with VMO moduli $\eta_{h}(r)$ such that $\eta_{h}(r) \leq \eta(r)$. In particular, for any $\varepsilon>0$ there exists a uniformly continuous function $g_{\varepsilon}$ on $\mathbf{R}^{N}$ such that $\left\|f-g_{\varepsilon}\right\|_{*}<\varepsilon$.

(ii) $V M O$ is a closed subspace of BMO and uniformly continuous functions that belong to BMO are VMO functions.

(iii) $W^{\theta, N / \theta}\left(\mathbf{R}^{N}\right) \subset \mathrm{VMO}$, for $0<\theta \leq 1$.

Example 2.1. (i) $\ln |x| \in$ BMO, but $\ln |x| \notin V M O$.

(ii) $\ln |\ln | x|| \in \mathrm{VMO}$.

\section{Feller semigroups and Markov processes}

This section provides a brief description of the basic definitions and results about a class of semigroups, Feller semigroups, associated with Markov processes in probability theory, which forms a functional analytic background for the proofs of Theorems 1.1 and 1.2 (see $[6,15])$.

\subsection{Markov transition functions}

First, we give the precise definition of a transition function which is adapted to our analysis. From the viewpoint of functional analysis, the transition function is something more convenient than the Markov process itself.

Let $(K, \rho)$ be a locally compact, separable metric space and $\mathcal{B}$ the $\sigma$-algebra of all Borel sets in $K$. A function $p_{t}(x, E)$, defined for all $t \geq 0, x \in K$ and $E \in \mathcal{B}$, is called a (temporally homogeneous) Markov transition function on $K$ if it satisfies the following four conditions:

(a) $p_{t}(x, \cdot)$ is a non-negative measure on $\mathcal{B}$ and $p_{t}(x, K) \leq 1$ for each $t \geq 0$ and $x \in K$.

(b) $p_{t}(\cdot, E)$ is a Borel measurable function for each $t \geq 0$ and $E \in \mathcal{B}$.

(c) $p_{0}(x,\{x\})=1$ for each $x \in K$. 
(d) (The Chapman-Kolmogorov equation) For any $t, s \geq 0, x \in K$ and $E \in \mathcal{B}$, we have

$$
p_{t+s}(x, E)=\int_{K} p_{t}(x, d y) p_{s}(y, E) .
$$

It should be emphasized that equation (3.1) expresses the idea that a transition from the position $x$ to the set $E$ in time $t+s$ is composed of a transition from $x$ to some position $y$ in time $t$, followed by a transition from $y$ to the set $E$ in the remaining time $s$; the latter transition has probability $p_{s}(y, E)$ which depends only on $y$.

We add a point $\partial$ to the locally compact space $K$ as the point at infinity if $K$ is not compact, and as an isolated point if $K$ is compact; so the space $K_{\partial}=K \cup\{\partial\}$ is compact.

Let $C(K)$ be the space of real-valued, bounded continuous functions $f$ on $K$. The space $C(K)$ is a Banach space with the supremum norm

$$
\|f\|=\sup _{x \in K}|f(x)| \text {. }
$$

We introduce a closed subspace of $C(K)$ as follows:

$$
C_{0}(K)=\left\{f \in C(K): \lim _{x \rightarrow \partial} f(x)=0\right\} .
$$

It should be noticed that the space $C_{0}(K)$ may be identified with the subspace of $C\left(K_{\partial}\right)$ which consists of all functions $f$ satisfying $f(\partial)=0$ :

$$
C_{0}(K)=\left\{f \in C\left(K_{\partial}\right): f(\partial)=0\right\} .
$$

\subsection{Feller semigroups}

We can associate with each Markov transition function $p_{t}(x, \cdot)$ by formula $(1.6)$ a family $\left\{T_{t}\right\}_{t \geq 0}$ of bounded linear operators acting on the space $C_{0}(K)$, and the Chapman-Kolmogorov equation (3.1) implies that this family $\left\{T_{t}\right\}_{t \geq 0}$ forms a semigroup.

A family $\left\{T_{t}\right\}_{t \geq 0}$ of bounded linear operators acting on $C_{0}(K)$ is called a Feller semigroup on $K$ if it satisfies the following three conditions:

(i) $T_{t+s}=T_{t} \cdot T_{s}, t, s \geq 0 ; T_{0}=I$.

(ii) The family $\left\{T_{t}\right\}$ is strongly continuous in $t$ for each $t \geq 0$ :

$$
\lim _{s \downarrow 0}\left\|T_{t+s} f-T_{t} f\right\|=0, \quad f \in C_{0}(K) .
$$

(iii) The family $\left\{T_{t}\right\}$ is non-negative and contractive on $C_{0}(K)$ :

$$
f \in C_{0}(K), 0 \leq f(x) \leq 1 \quad \text { on } K \Longrightarrow 0 \leq T_{t} f(x) \leq 1 \quad \text { on } K .
$$

If $\left\{T_{t}\right\}_{t \geq 0}$ is a Feller semigroup on $K$, we define its infinitesimal generator $\mathfrak{A}$ by the formula

$$
\mathfrak{A} u=\lim _{t \downarrow 0} \frac{T_{t} u-u}{t},
$$

provided that the limit (3.3) exists in $C_{0}(K)$. More precisely, the infinitesimal generator $\mathfrak{A}$ is a linear operator from the space $C_{0}(K)$ into itself defined as follows. 
(1) The domain $D(\mathfrak{A})$ is the set

$$
D(\mathfrak{A})=\left\{u \in C_{0}(K) \text { : the limit (3.3) exists }\right\} .
$$

(2) $\mathfrak{A} u=\lim _{t \downarrow 0} \frac{T_{t} u-u}{t}, u \in D(\mathfrak{A})$.

The next theorem is a version of the Hille-Yosida theorem [16] adapted to the present context (see [6, Theorem 9.3.1 and Corollary 9.3.2]):

Theorem 3.1. (i) Let $\left\{T_{t}\right\}_{t \geq 0}$ be a Feller semigroup on $K$ and $\mathfrak{A}$ its infinitesimal generator. Then we have the following four assertions:

(a) The domain $D(\mathfrak{A})$ is dense in the space $C_{0}(K)$.

(b) For each $\alpha>0$, the equation $(\alpha I-\mathfrak{A}) u=f$ has a unique solution $u$ in $D(\mathfrak{A})$ for any $f \in C_{0}(K)$. Hence, for each $\alpha>0$, the Green operator $(\alpha I-\mathfrak{A})^{-1}: C_{0}(K) \rightarrow C_{0}(K)$ can be defined by the formula

$$
u=(\alpha I-\mathfrak{A})^{-1} f, \quad f \in C_{0}(K)
$$

(c) For each $\alpha>0$, the operator $(\alpha I-\mathfrak{A})^{-1}$ is non-negative on the space $C_{0}(K)$ :

$$
f \in C_{0}(K), f \geq 0 \quad \text { on } K \Longrightarrow(\alpha I-\mathfrak{A})^{-1} f \geq 0 \quad \text { on } K .
$$

(d) For each $\alpha>0$, the operator $(\alpha I-\mathfrak{A})^{-1}$ is bounded on the space $C_{0}(K)$ with norm

$$
\left\|(\alpha I-\mathfrak{A})^{-1}\right\| \leq \frac{1}{\alpha} .
$$

(ii) Conversely, if $\mathfrak{A}$ is a linear operator from $C_{0}(K)$ into itself satisfying condition (a) and if there is a constant $\alpha_{0} \geq 0$ such that, for all $\alpha>$ $\alpha_{0}$, conditions (b) through (d) are satisfied, then $\mathfrak{A}$ is the infinitesimal generator of some Feller semigroup $\left\{T_{t}\right\}_{t \geq 0}$ on $K$.

\section{The Dirichlet problem}

In this section we consider the Dirichlet problem for the diffusion operator $A$ in the framework of Sobolev spaces of $L^{p}$ style, and prove an existence and uniqueness theorem for the Dirichlet problem with VMO coefficients.

\subsection{Formulation of a boundary value problem}

If $1<p<\infty$, we define the Sobolev space

$$
\begin{aligned}
W^{2, p}(\Omega)= & \text { the space of (equivalence classes of) functions } \\
& u \in L^{p}(\Omega) \text { whose derivatives } D^{\alpha} u,|\alpha| \leq 2 \text {, in the } \\
& \text { sense of distributions are in } L^{p}(\Omega),
\end{aligned}
$$

and the boundary space

$$
\begin{aligned}
B^{2-1 / p, p}(\partial \Omega)= & \text { the space of the boundary values } \gamma_{0} u \text { of functions } \\
& u \in W^{2, p}(\Omega) .
\end{aligned}
$$


In the boundary space $B^{2-1 / p, p}(\partial \Omega)$, we introduce a norm

$$
|\varphi|_{B^{2-1 / p, p}(\partial \Omega)}=\inf \left\{\|u\|_{W^{2, p}(\Omega)}: u \in W^{2, p}(\Omega), \gamma_{0} u=\varphi \text { on } \partial \Omega\right\} .
$$

It is known (see [17-19]) that the space $B^{2-1 / p, p}(\partial \Omega)$ is a Besov space.

We consider the following non-homogeneous Dirichlet boundary value problem: Given functions $f$ and $\varphi$ defined in $\Omega$ and on $\partial \Omega$, respectively, find a function $u$ in $\Omega$ such that

$$
\begin{cases}A u=f & \text { in } \Omega \\ \gamma_{0} u=\varphi & \text { on } \partial \Omega\end{cases}
$$

In this framework our main result is a generalization of Bony [10, Théorème 3] to the VMO case:

Theorem 4.1. Let $N<p<\infty$. Then the mapping

$$
\mathcal{A}=\left(A, \gamma_{0}\right): W^{2, p}(\Omega) \longrightarrow L^{p}(\Omega) \bigoplus B^{2-1 / p, p}(\partial \Omega)
$$

is an algebraic and topological isomorphism. In particular, for any $f \in L^{p}(\Omega)$ and any $\varphi \in B^{2-1 / p, p}(\partial \Omega)$, there exists a unique solution $u \in W^{2, p}(\Omega)$ of problem (4.1).

Remark 4.1. Theorem 4.1 will play an essential role in the study of the existence of positive solutions of semilinear Dirichlet eigenvalue problems for diffusive logistic equations with discontinuous coefficients which model population dynamics in environments with spatial heterogeneity (see [20, Theorem 1.1]).

\subsection{Proof of Theorem 4.1}

In this subsection we prove Theorem 4.1. To do this, it suffices to show that the mapping

$$
\mathcal{A}=\left(A, \gamma_{0}\right): W^{2, p}(\Omega) \longrightarrow L^{p}(\Omega) \bigoplus B^{2-1 / p, p}(\partial \Omega)
$$

is bijective. Indeed, the continuity of the inverse of $\mathcal{A}$ follows immediately from an application of Banach's closed graph theorem.

The proof is divided into four steps.

Step 1: Our proof of Theorem 4.1 is essentially based on the following existence and uniqueness theorem for the homogeneous Dirichlet problem due to Chiarenza-Frasca-Longo [21, Theorems 4.3 and 4.4] (see also [22, 23]):

Theorem 4.2. If we let

$$
A_{0} u=\sum_{i, j=1}^{N} a^{i j}(x) \frac{\partial^{2} u}{\partial x_{i} \partial x_{j}}
$$

then, for any $f \in L^{p}(\Omega)$ with $1<p<\infty$ there exists a unique solution $u \in$ $W^{2, p}(\Omega)$ of the Dirichlet problem

$$
\begin{cases}A_{0} u=f & \text { in } \Omega, \\ \gamma_{0} u=0 & \text { on } \partial \Omega .\end{cases}
$$


Now, for any $\varphi \in B^{2-1 / p, p}(\partial \Omega)$, we can find a function $v \in W^{2, p}(\Omega)$ such that $\gamma_{0} v=\varphi$ on $\partial \Omega$. Hence we obtain the following existence and uniqueness theorem for the non-homogeneous Dirichlet problem:

Corollary 4.3. Let $1<p<\infty$. For any $f \in L^{p}(\Omega)$ and any $\varphi \in B^{2-1 / p, p}(\partial \Omega)$, there exists a unique solution $u \in W^{2, p}(\Omega)$ of the Dirichlet problem

$$
\begin{cases}A_{0} u=f & \text { in } \Omega, \\ \gamma_{0} u=\varphi & \text { on } \partial \Omega .\end{cases}
$$

If we associate with problem (4.3) a continuous linear operator

$$
\mathcal{A}_{0}=\left(A_{0}, \gamma_{0}\right): W^{2, p}(\Omega) \longrightarrow L^{p}(\Omega) \bigoplus B^{2-1 / p, p}(\partial \Omega),
$$

then Corollary 4.3 asserts that the mapping $\mathcal{A}_{0}$ is an algebraic and topological isomorphism. In particular, we have, for $1<p<\infty$,

$$
\text { ind } \mathcal{A}_{0}=0 \text {. }
$$

Step 2: If we let

$$
B u=\sum_{i=1}^{N} b^{i}(x) \frac{\partial u}{\partial x_{i}}+c(x) u,
$$

then it is clear that the operator

$$
B: W^{2, p}(\Omega) \longrightarrow W^{1, p}(\Omega)
$$

is continuous. Moreover, it follows from an application of the Rellich-Kondrachov theorem (see $[24$, Theorem 7.26$]$ ) that the injection $W^{1, p}(\Omega) \rightarrow L^{p}(\Omega)$ is compact. Hence we find that the mapping

$$
B: W^{2, p}(\Omega) \longrightarrow L^{p}(\Omega)
$$

is compact.

Therefore, we obtain that the mapping

$$
\mathcal{A}=\left(A_{0}+B, \gamma_{0}\right)=\mathcal{A}_{0}+(B, 0): W^{2, p}(\Omega) \longrightarrow L^{p}(\Omega) \bigoplus B^{2-1 / p, p}(\partial \Omega)
$$

is a Fredholm operator with index zero, since we have, by assertion (4.4),

$$
\text { ind } \mathcal{A}=\text { ind } \mathcal{A}_{0}=0 \text {. }
$$

Step 3: On the other hand, the uniqueness result in Theorem 4.1 follows from a variant of the Bakel'man-Aleksandrov maximum principle in the framework of Sobolev spaces due to Bony [10, Théorème 2] (see also [25, Lemma 3.25]):

Theorem 4.4. Let $N<p<\infty$. If a function $u \in W^{2, p}(\Omega)$ satisfies the condition

$$
A u(x) \geq 0 \quad \text { for almost all } x \in \Omega,
$$

then it follows that

$$
\max _{\bar{\Omega}} u \leq \max _{\partial \Omega} u^{+}
$$

where

$$
u^{+}(x)=\max \{u(x), 0\}, \quad x \in \bar{\Omega} .
$$


Remark 4.2. In fact, Bony proved this maximum principle under the weaker condition that $a^{i j} \in L^{\infty}(\Omega)$ (see the proof of [10, Théorème 1]).

Here it should be noticed that we have, by Sobolev's imbedding theorem,

$$
W^{2, p}(\Omega) \subset C^{1}(\bar{\Omega}),
$$

since $N<p<\infty$ and so $2-N / p>1$. By applying Theorem 4.4 to the functions $\pm u(x)$, we find that

$$
\begin{cases}A u=0 & \text { almost everywhere in } \Omega, \quad \Longrightarrow \quad u=0 \quad \text { in } \Omega . \\ \gamma_{0} u=0 & \text { on } \partial \Omega\end{cases}
$$

This implies that the mapping

$$
\mathcal{A}=\left(A, \gamma_{0}\right): W^{2, p}(\Omega) \longrightarrow L^{p}(\Omega) \bigoplus B^{2-1 / p, p}(\partial \Omega)
$$

is injective for $N<p<\infty$. Hence it is also surjective for $N<p<\infty$, since we have ind $\mathcal{A}=0$ for $1<p<\infty$.

Step 4: Summing up, we have proved that the mapping

$$
\mathcal{A}=\left(A, \gamma_{0}\right): W^{2, p}(\Omega) \longrightarrow L^{p}(\Omega) \bigoplus B^{2-1 / p, p}(\partial \Omega)
$$

is an algebraic and topological isomorphism for $N<p<\infty$.

Now the proof of Theorem 4.1 is complete.

\section{$5 \quad$ Proof of Theorem 1.1}

In this section we consider the Dirichlet problem for the Waldenfels operator $W=A+S$ in the framework of Sobolev spaces of $L^{p}$ style, and prove Theorem 1.1. The essential point in the proof is how to estimate the Lévy integral operator

$$
S u(x)=\int_{\bar{\Omega}} s(x, d y)\left[u(y)-u(x)-\sum_{j=1}^{N} \frac{\partial u}{\partial x_{j}}(x)\left(y_{j}-x_{j}\right)\right]
$$

in terms of Sobolev norms. We show that the operator $\mathcal{W}=\left(W, \gamma_{0}\right)$ may be considered as a perturbation of a compact operator to the operator $\mathcal{A}=\left(A, \gamma_{0}\right)$ in the framework of Sobolev spaces. Therefore, the proof of Theorem 1.1 is reduced to the differential operator case which is studied in Section 4.

The proof is divided into three steps.

Step 1: First, we study the integral operator $S$ in the framework of Sobolev spaces. To do this, we need the following lemma from real analysis (see $[2$, Lemme 1]):

Lemma 5.1. If $u \in W^{2, p}\left(\mathbf{R}^{N}\right)$ with $N<p<\infty$, we let

$$
U(x)=\sup _{\substack{t \in \mathbf{R}^{N} \\ t \neq 0}} \frac{\left|u(x+t)-u(x)-\sum_{j=1}^{N} \frac{\partial u}{\partial x_{j}}(x) t_{j}\right|}{|t|^{2}}, \quad x \in \mathbf{R}^{N} .
$$

Then it follows that $U \in L^{p}\left(\mathbf{R}^{N}\right)$. More precisely, we have the estimate

$$
\|U\|_{L^{p}\left(\mathbf{R}^{N}\right)} \leq C_{1}\|u\|_{W^{2, p}\left(\mathbf{R}^{N}\right)},
$$

with some constant $C_{1}>0$ depending on $N$ and $p$. 
By virtue of Lemma 5.1, we can estimate the integral term $S u$ in terms of Sobolev norms, just as in Bony [2, Théorème 1]:

Lemma 5.2. For every $\varepsilon>0$, there exist constants $c_{0}>0$ and $C_{1}>0$, independent of $\varepsilon$, such that we have, for all $u \in W^{2, p}(\Omega)$,

$$
\|S u\|_{L^{p}(\Omega)} \leq c_{0} \omega(\varepsilon)\|u\|_{W^{2, p}(\Omega)}+C_{\varepsilon}\left(1+\frac{C_{1}}{\varepsilon^{N / p}}\right)\|\nabla u\|_{L^{p}(\Omega)} .
$$

Here

$$
C_{\varepsilon}=\sup _{x \in \Omega} \int_{\{y \in \bar{\Omega}:|y-x|>\varepsilon\}} s(x, d y)|y-x| .
$$

Step 2: We have, by Theorem 4.1,

$$
\text { ind } \mathcal{A}=\operatorname{ind}\left(A, \gamma_{0}\right)=0
$$

On the other hand, inequality (5.1) tells us that the integral operator $S$ is a compact operator from $W^{2, p}(\Omega)$ into $L^{p}(\Omega)$, since $\lim _{\varepsilon \downarrow 0} \omega(\varepsilon)=0$.

Therefore, we obtain that the operator

$$
\mathcal{W}=\left(A+S, \gamma_{0}\right)=\left(A, \gamma_{0}\right)+(S, 0): W^{2, p}(\Omega) \longrightarrow L^{p}(\Omega) \bigoplus B^{2-1 / p, p}(\partial \Omega)
$$

is a perturbation of a compact operator to the operator $\mathcal{A}=\left(A, \gamma_{0}\right)$. In particular, we have

$$
\text { ind } \mathcal{W}=\text { ind } \mathcal{A}=0 \text {. }
$$

Step 3: Hence, in order to show the bijectivity of $\mathcal{W}=\left(W, \gamma_{0}\right)$ it suffices to prove its injectivity:

$$
\left\{\begin{array}{l}
u \in W^{2, p}(\Omega), W u=0 \text { in } \Omega, \gamma_{0} u=0 \text { on } \partial \Omega \\
\Longrightarrow u=0 \text { in } \Omega .
\end{array}\right.
$$

However, this is an immediate consequence of the following maximum principle due to Bony [2, Théorème 3]):

Theorem 5.3. Let $N<p<\infty$. If a function $u \in W^{2, p}(\Omega)$ satisfies the condition

$$
W u(x) \geq 0 \quad \text { for almost all } x \in \Omega,
$$

then it follows that the function $u(x)$ may take its positive maximum only on the boundary $\partial \Omega$.

Remark 5.1. It should be emphasized that Bony proved this maximum principle under the weaker condition that $a^{i j} \in L^{\infty}(\Omega)$ (see the proof of $[10$, Théorème 1]).

Indeed, the desired assertion (5.3) follows by applying Theorem 5.3 to the functions $\pm u(x)$.

Step 4: Finally, the continuity of the inverse of $\mathcal{W}$ follows immediately from an application of Banach's closed graph theorem.

The proof of Theorem 1.1 is now complete. 


\section{Proof of Theorem 1.2}

In this section we apply a version of the Hille-Yosida theorem (Theorem 3.1) to prove Theorem 1.2. We make use of an $L^{\infty}$ estimate for the Green operator $G_{\alpha}^{0}$ of the Dirichlet problem to verify all the conditions in Theorem 3.1 for the operator $\mathfrak{W}$.

\subsection{The space $C_{0}(\bar{\Omega})$}

First, we consider a one-point compactification $K_{\partial}=K \cup\{\partial\}$ of the locally compact space $K=\Omega$. We say that two points $x$ and $y$ of $\bar{\Omega}$ are equivalent modulo $\partial \Omega$ if either $x=y$ or $x, y \in \partial \Omega$. We denote by $\bar{\Omega} / \partial \Omega$ the totality of equivalence classes modulo $\partial \Omega$. On the set $\bar{\Omega} / \partial \Omega$ we define the quotient topology induced by the projection

$$
q: \bar{\Omega} \longrightarrow \bar{\Omega} / \partial \Omega \text {. }
$$

Then it is easy to see that the topological space $\bar{\Omega} / \partial \Omega$ is a one-point compactification $K_{\partial}$ of the space $\Omega$ and that the point at infinity $\partial$ corresponds to the boundary $\partial \Omega$ :

$$
K_{\partial}:=\bar{\Omega} / \partial \Omega, \quad \partial:=\partial \Omega .
$$

Furthermore, we have the following two assertions:

(i) If $\tilde{u}$ is a continuous function defined on $\bar{\Omega} / \partial \Omega$, then the function $\tilde{u} \circ q$ is continuous on $\bar{\Omega}$ and constant on $\partial \Omega$.

(ii) Conversely, if $u$ is a continuous function defined on $\bar{\Omega}$ and constant on $\partial \Omega$, then it defines a continuous function $\tilde{u}$ on $\bar{\Omega} / \partial \Omega$.

In other words, we have the isomorphism

$$
C\left(K_{\partial}\right) \cong\{u \in C(\bar{\Omega}): u \text { is constant on } \partial \Omega\} .
$$

Now we introduce a closed subspace of $C\left(K_{\partial}\right)$ as follows (cf. formula (3.2)):

$$
C_{0}(K)=\left\{u \in C\left(K_{\partial}\right): u(\partial)=0\right\} .
$$

Then we have, by assertion (6.1), the isomorphism

$$
C_{0}(K) \cong C_{0}(\bar{\Omega})=\{u \in C(\bar{\Omega}): u=0 \text { on } \partial \Omega\} .
$$

\subsection{Proof of Theorem 1.2}

In order to prove Theorem 1.2, it suffices to verify all conditions (a) through (d) in Theorem 3.1 for the operator $\mathfrak{W}$. The proof is divided into four steps.

Step 1: (b) For each $\alpha>0$, the equation $(\alpha I-\mathfrak{W}) u=f$ has a unique solution $u \in D(\mathfrak{W})$ for any $f \in C_{0}(\bar{\Omega})$.

Since we have

$$
c(x)-\alpha \leq-\alpha \text { for almost all } x \in \Omega,
$$

applying Theorem 1.1 to the operator $W-\alpha$ we obtain that the Dirichlet problem

$$
\begin{cases}(\alpha-W) u=f & \text { almost everywhere in } \Omega \\ \gamma_{0} u=0 & \text { on } \partial \Omega\end{cases}
$$


has a unique solution $u \in W^{2, p}(\Omega)$ for any $f \in L^{p}(\Omega)$ with $N<p<\infty$. In particular, for any $f \in C_{0}(\bar{\Omega})$ there exists a function $u \in W^{2, p}(\Omega) \cap C_{0}(\bar{\Omega})$ such that

$$
(\alpha-W) u=f \quad \text { in } \Omega .
$$

Hence we have

$$
W u=\alpha u-f \in C_{0}(\bar{\Omega}) .
$$

This proves that

$$
\left\{\begin{array}{l}
u \in D(\mathfrak{W}) \\
(\alpha I-\mathfrak{W}) u=f .
\end{array}\right.
$$

Step 2: (c) For each $\alpha>0$, the Green operator $G_{\alpha}^{0}=(\alpha I-\mathfrak{W})^{-1}$ is non-negative on the space $C_{0}(\bar{\Omega})$ :

$$
f \in C_{0}(\bar{\Omega}), f(x) \geq 0 \quad \text { in } \Omega \Longrightarrow u(x)=G_{\alpha}^{0} f(x) \geq 0 \quad \text { in } \Omega .
$$

Indeed, if we let

$$
v(x)=-u(x)=-G_{\alpha}^{0} f(x),
$$

then it follows that

$$
\begin{cases}(W-\alpha) v=f \geq 0 & \text { in } \Omega, \\ \gamma_{0} v=0 & \text { on } \partial \Omega .\end{cases}
$$

Therefore, applying Theorem 5.3 to the operator $W-\alpha$ with $\alpha>0$ we obtain that

$$
v(x) \leq 0 \quad \text { in } \Omega,
$$

so that

$$
u(x)=-v(x) \geq 0 \quad \text { in } \Omega .
$$

Step 3: (d) For each $\alpha>0$, the Green operator $G_{\alpha}^{0}=(\alpha I-\mathfrak{W})^{-1}$ is bounded on the space $C_{0}(\bar{\Omega})$ with norm $1 / \alpha$ : $\left\|G_{\alpha}^{0}\right\| \leq 1 / \alpha$.

Let $f(x)$ be an arbitrary function in $C_{0}(\bar{\Omega})$. If we let

$$
u_{ \pm}(x)= \pm \alpha G_{\alpha}^{0} f(x)-\|f\| \in W^{2, p}(\Omega),
$$

we have only to prove that

$$
u_{ \pm}(x) \leq 0 \quad \text { in } \Omega
$$

Indeed, it follows that

$$
\begin{aligned}
(W-\alpha) u_{ \pm}(x) & =\mp \alpha f(x)+(\alpha-c(x))\|f\| \\
& =\alpha(\|f\| \mp f(x))+(-c(x))\|f\| \\
& \geq 0 \text { in } \Omega .
\end{aligned}
$$

Thus, applying Theorem 5.3 to the operator $W-\alpha$ with $\alpha>0$ we obtain that the function $u_{ \pm}(x)$ may take its positive maximum only on $\partial \Omega$. This proves assertion (6.2), since we have

$$
\gamma_{0}\left(u_{ \pm}\right)=-\|f\| \leq 0 \quad \text { on } \partial \Omega .
$$

Step 4: (a) The domain $D(\mathfrak{W})$ is dense in $C_{0}(\bar{\Omega})$. More precisely, we prove that, for each $u \in C_{0}(\bar{\Omega})$, we have the assertion

$$
\lim _{\alpha \rightarrow+\infty}\left\|\alpha G_{\alpha}^{0} u-u\right\|=0 .
$$


Since the space

$$
C_{0}^{2}(\bar{\Omega}):=C^{2}(\bar{\Omega}) \cap C_{0}(\bar{\Omega})
$$

is dense in $C_{0}(\bar{\Omega})$, it suffices to prove assertion (6.3) for any $u \in C_{0}^{2}(\bar{\Omega})$.

Step 4-1: To prove assertion (6.3) for any $u \in C_{0}^{2}(\bar{\Omega})$, we shall introduce an extension $\widetilde{G_{\alpha}^{0}}$ of the Green operator $G_{\alpha}^{0}$ to the space $L^{\infty}(\Omega)$ :

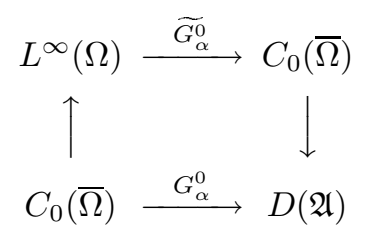

By applying Theorem 1.1 the operator $W-\alpha$ with $\alpha>0$, we find that the Dirichlet problem

$$
\begin{cases}(\alpha-W) u=f & \text { almost everywhere in } \Omega \\ \gamma_{0} u=0 & \text { on } \partial \Omega\end{cases}
$$

has a unique solution $u \in W^{2, p}(\Omega) \cap C_{0}(\bar{\Omega})$ for any $f \in L^{\infty}(\Omega)$. If we let

$$
u=\widetilde{G_{\alpha}^{0}} f
$$

then it is easy to verify that the operator $\widetilde{G_{\alpha}^{0}}$ is an extension of $G_{\alpha}^{0}$ to $L^{\infty}(\Omega)$. Moreover, just as in Steps 2 and 3, we can prove the following two assertions:

(A) The operator $\widetilde{G_{\alpha}^{0}}: L^{\infty}(\Omega) \rightarrow C_{0}(\bar{\Omega})$ is non-negative.

(B) The operator $\widetilde{G_{\alpha}^{0}}: L^{\infty}(\Omega) \rightarrow C_{0}(\bar{\Omega})$ is bounded with norm $1 / \alpha$ : $\left\|\widetilde{G_{\alpha}^{0}}\right\| \leq$ $1 / \alpha$.

Step 4-2: First, since all the coefficients of the operator $A$ belong to $L^{\infty}(\Omega)$, it follows that

$$
\begin{gathered}
A u=\sum_{i, j=1}^{N} a^{i j}(x) \frac{\partial^{2} u}{\partial x_{i} \partial x_{j}}+\sum_{i=1}^{N} b^{i}(x) \frac{\partial u}{\partial x_{i}}+c(x) u \in L^{\infty}(\Omega), \\
u \in C_{0}^{2}(\bar{\Omega}) .
\end{gathered}
$$

Furthermore, we can obtain an $L^{\infty}$-version of Lemma 5.2 as follows:

Lemma 6.1. For every $\varepsilon>0$, there exists a constant $C_{2}>0$, independent of $\varepsilon$, such that we have, for all $u \in C_{0}^{2}(\bar{\Omega})$,

$$
\|S u\|_{L^{\infty}(\Omega)} \leq \frac{1}{2} \omega(\varepsilon)\left\|\nabla^{2} u\right\|_{L^{\infty}(\Omega)}+C_{\varepsilon}\left(1+\frac{C_{2}}{\varepsilon^{N / p}}\right)\|\nabla u\|_{L^{\infty}(\Omega)} .
$$

Proof. For each $\varepsilon>0$, we decompose the integral term $S u(x)$ into the two terms $S_{\varepsilon}^{(1)} u$ and $S_{\varepsilon}^{(2)} u$ as follows:

$$
\begin{aligned}
S u(x)= & \int_{\{y \in \bar{\Omega}:|y-x| \leq \varepsilon\}} s(x, d y)\left[u(y)-u(x)-\sum_{j=1}^{N} \frac{\partial u}{\partial x_{j}}(x)\left(y_{j}-x_{j}\right)\right] \\
& +\int_{\{y \in \bar{\Omega}:|y-x|>\varepsilon\}} s(x, d y)\left[u(y)-u(x)-\sum_{j=1}^{N} \frac{\partial u}{\partial x_{j}}(x)\left(y_{j}-x_{j}\right)\right] \\
:= & S_{\varepsilon}^{(1)} u(x)+S_{\varepsilon}^{(2)} u(x) .
\end{aligned}
$$


(1) First, we have, by Taylor's formula,

$$
\begin{aligned}
& \left|S_{\varepsilon}^{(1)} u(x)\right| \\
& \quad \leq \int_{\{y \in \bar{\Omega}: 0<|y-x| \leq \varepsilon\}} s(x, d y)\left|u(y)-u(x)-\sum_{j=1}^{N} \frac{\partial u}{\partial x_{j}}(x)\left(y_{j}-x_{j}\right)\right| \\
& \quad=\int_{\{y \in \bar{\Omega}: 0<|y-x| \leq \varepsilon\}} s(x, d y)|y-x|^{2} \cdot \frac{\left|u(y)-u(x)-\sum_{j=1}^{N} \frac{\partial u}{\partial x_{j}}(x)\left(y_{j}-x_{j}\right)\right|}{|y-x|^{2}} \\
& \quad \leq \frac{1}{2} \int_{\{y \in \bar{\Omega}: 0<|y-x| \leq \varepsilon\}} s(x, d y)|y-x|^{2} \cdot\left\|\nabla^{2} u\right\|_{L^{\infty}(\Omega)} \\
& \quad \leq \frac{1}{2} \omega(\varepsilon)\left\|\nabla^{2} u\right\|_{L^{\infty}(\Omega)},
\end{aligned}
$$

so that

$$
\left\|S_{\varepsilon}^{(1)} u\right\|_{L^{\infty}(\Omega)} \leq \frac{1}{2} \omega(\varepsilon)\left\|\nabla^{2} u\right\|_{L^{\infty}(\Omega)} .
$$

(2) Secondly, we rewrite the term $S_{\varepsilon}^{(2)} u$ in the form

$$
\begin{aligned}
S_{\varepsilon}^{(2)} u(x)= & \int_{\{y \in \bar{\Omega}:|y-x|>\varepsilon\}} s(x, d y)[u(y)-u(x)] \\
& +\sum_{j=1}^{N} \int_{\{y \in \bar{\Omega}:|y-x|>\varepsilon\}} s(x, d y) \frac{\partial u}{\partial x_{j}}(x)\left(y_{j}-x_{j}\right) \\
:= & A(x)+B(x) .
\end{aligned}
$$

Then, by using condition (1.4) we can estimate the term $B(x)$ as follows:

$$
|B(x)| \leq \int_{\{y \in \bar{\Omega}:|y-x|>\varepsilon\}} s(x, d y)|y-x| \cdot \sum_{j=1}^{N}\left|\frac{\partial u}{\partial x_{j}}(x)\right| \leq C_{\varepsilon}|\nabla u(x)| .
$$

Hence we have

$$
\|B\|_{L^{\infty}(\Omega)} \leq C_{\varepsilon}\|\nabla u\|_{L^{\infty}(\Omega)} .
$$

On the other hand, by Morrey's imbedding theorem (see [24, Theorem 7.17]) we can find a constant $C>0$ such that

$$
|u(y)-u(x)| \leq C|y-x|^{1-N / p}\|\nabla u\|_{L^{p}(\Omega)} .
$$

Hence it follows that

$$
\begin{aligned}
|A(x)| & \leq \int_{\{y \in \bar{\Omega}:|y-x|>\varepsilon\}} s(x, d y)|u(y)-u(x)| \\
& \leq C \int_{\{y \in \bar{\Omega}:|y-x|>\varepsilon\}} s(x, d y)|y-x|^{1-N / p} \cdot\|\nabla u\|_{L^{p}(\Omega)} \\
& =C \int_{\{y \in \bar{\Omega}:|y-x|>\varepsilon\}} s(x, d y)|y-x| \cdot \frac{1}{|y-x|^{N / p}}\|\nabla u\|_{L^{p}(\Omega)} \\
& \leq \frac{C}{\varepsilon^{N / p}}\left(\int_{\{y \in \bar{\Omega}:|y-x|>\varepsilon\}} s(x, d y)|x-y|\right)\|\nabla u\|_{L^{p}(\Omega)} \\
& \leq \frac{C C_{\varepsilon}}{\varepsilon^{N / p}}\|\nabla u\|_{L^{p}(\Omega)} .
\end{aligned}
$$


This proves that

$$
\|A\|_{L^{\infty}(\Omega)} \leq \frac{C C_{\varepsilon}}{\varepsilon^{N / p}}\|\nabla u\|_{L^{p}(\Omega)} \leq \frac{C C_{\varepsilon}}{\varepsilon^{N / p}}|\Omega|^{1 / p}\|\nabla u\|_{L^{\infty}(\Omega)},
$$

where $|\Omega|$ is the volume of the domain $\Omega$.

By estimates (6.7) and (6.8), it follows that

$$
\begin{aligned}
\left\|S_{\varepsilon}^{(2)} u\right\|_{L^{\infty}(\Omega)} & \leq\|A\|_{L^{\infty}(\Omega)}+\|B\|_{L^{\infty}(\Omega)} \\
& \leq C_{\varepsilon}\left(1+\frac{C|\Omega|^{1 / p}}{\varepsilon^{N / p}}\right)\|\nabla u\|_{L^{\infty}(\Omega)} .
\end{aligned}
$$

(3) The desired estimate (6.5) follows by combining estimates (6.6) and (6.9):

$$
\begin{aligned}
\|S u\|_{L^{\infty}(\Omega)} & \leq\left\|S_{\varepsilon}^{(1)} u\right\|_{L^{\infty}(\Omega)}+\left\|S_{\varepsilon}^{(2)} u\right\|_{L^{\infty}(\Omega)} \\
& \leq \frac{1}{2} \omega(\varepsilon)\left\|\nabla^{2} u\right\|_{L^{\infty}(\Omega)}+C_{\varepsilon}\left(1+\frac{C_{2}}{\varepsilon^{N / p}}\right)\|\nabla u\|_{L^{\infty}(\Omega)},
\end{aligned}
$$

with

$$
C_{2}:=C|\Omega|^{1 / p} .
$$

The proof of Lemma 6.1 is complete.

Therefore, we obtain from assertion (6.4) and estimate (6.5) that

$$
W u=A u+S u \in L^{\infty}(\Omega), \quad u \in C_{0}^{2}(\bar{\Omega}) .
$$

Step 4-3: Now, if we let

$$
w=\alpha G_{\alpha}^{0} u-\widetilde{G_{\alpha}^{0}}(W u)
$$

then we have

$$
\left\{\begin{array}{l}
w \in W^{2, p}(\Omega) \cap C_{0}(\bar{\Omega}) \\
(W-\alpha) w=-\alpha u+W u=(W-\alpha) u \quad \text { in } \Omega
\end{array}\right.
$$

and so

$$
\left\{\begin{array}{l}
w-u \in W^{2, p}(\Omega) \cap C_{0}(\bar{\Omega}) \\
(W-\alpha)(w-u)=0 \quad \text { in } \Omega .
\end{array}\right.
$$

By Theorem 1.1, this implies that $w-u=0$ in $\Omega$, that is,

$$
u=w=\alpha G_{\alpha}^{0} u-\widetilde{G_{\alpha}^{0}}(W u) .
$$

Therefore, the desired assertion (6.3) for any $u \in C_{0}^{2}(\bar{\Omega})$ follows from an application of assertion (B), since we have, for all $\alpha>0$,

$$
\left\|u-\alpha G_{\alpha}^{0} u\right\|=\left\|\widetilde{G_{\alpha}^{0}}(W u)\right\|_{L^{\infty}(\Omega)} \leq \frac{1}{\alpha}\|W u\|_{L^{\infty}(\Omega)} .
$$

Now the proof of Theorem 1.2 is complete. 


\subsection{Proof of Remark 1.3}

Finally, we prove that the domain

$$
\mathcal{D}(\mathfrak{W})=\left\{u \in C_{0}(\bar{\Omega}) \cap W^{2, p}(\Omega): W u \in C_{0}(\bar{\Omega})\right\}
$$

is independent of $N<p<\infty$ (cf. the proof of [26, Lemma 4.2]).

We let

$$
\mathcal{D}_{p}:=\left\{u \in W^{2, p}(\Omega) \cap C_{0}(\bar{\Omega}): W u \in C_{0}(\bar{\Omega})\right\} .
$$

In order to prove Remark 1.3, it suffices to show that

$$
\mathcal{D}_{p_{1}}=\mathcal{D}_{p_{2}} \quad \text { for } N<p_{1}<p_{2}<\infty .
$$

First, it follows that

$$
\mathcal{D}_{p_{2}} \subset \mathcal{D}_{p_{1}}
$$

since we have $L^{p_{2}}(\Omega) \subset L^{p_{1}}(\Omega)$ for $p_{2}>p_{1}$.

Conversely, let $v$ be an arbitrary element of $\mathcal{D}_{p_{1}}$ :

$$
v \in W^{2, p_{1}}(\Omega) \cap C_{0}(\bar{\Omega}), \quad W v \in C_{0}(\bar{\Omega}) .
$$

Then, since we have $v, W v \in C_{0}(\bar{\Omega}) \subset L^{p_{2}}(\Omega)$, it follows from an application of Theorem 1.1 with $p:=p_{2}$ that there exists a unique function $u \in W^{2, p_{2}}(\Omega)$ such that

$$
\begin{cases}W u=W v & \text { in } \Omega \\ \gamma_{0} u=0 & \text { on } \partial \Omega\end{cases}
$$

Hence we have

$$
\begin{cases}u-v \in W^{2, p_{1}}(\Omega), & \\ W(u-v)=0 & \text { in } \Omega, \\ \gamma_{0}(u-v)=0 & \text { on } \partial \Omega .\end{cases}
$$

Therefore, by applying again Theorem 1.1 with $p:=p_{1}$ we obtain that $u-v=0$, so that $v=u \in W^{2, p_{2}}(\Omega)$. This implies that $v \in \mathcal{D}_{p_{2}}$.

\section{Acknowledgements}

The author is grateful to the referee for many valuable suggestions which improved the presentation of this paper.

\section{References}

[1] Taira, K., Semigroups, boundary value problems and Markov processes, Springer-Verlag, Berlin Heidelberg New York, 2004.

[2] Bony, J.-M., Problème de Dirichlet et semi-groupe fortement fellérien associés à un opérateur intégro-différentiel, C. R. Acad. Sc. Paris 265, 361364 (1967).

[3] Gimbert, F., Lions, P. L., Existence and regularity results for solutions of second-order, elliptic, integrodifferential operators, Ricerche di Matematica 33, 315-358 (1984). 
[4] Garroni, M. G. and Menaldi, J. L., Second order elliptic integro-differential problems, Research Notes in Mathematics Series No. 430, Chapman \& Hall/CRC, Boca Raton London New York Washington, D.C., 2002.

[5] Dynkin, E. B., Markov processes I, II, Springer-Verlag, Berlin, Göttingen, Heidelberg, 1965.

[6] Taira, K., Diffusion processes and partial differential equations, Academic Press, San Diego New York London Tokyo, 1988.

[7] Komatsu, T., Markov processes associated with certain integro-differential operators, Osaka J. Math. 10, 271-303 (1973).

[8] Stroock, D. W., Diffusion processes associated with Lévy generators, Z. Wahrscheinlichkeitstheorie verw. Gebiete 32, 209-244 (1975).

[9] Garroni, M. G. and Menaldi, J. L., Green functions for second order integro-differential problems, Pitman Research Notes in Mathematics Series No. 275, Longman Scientific \& Technical, Harlow, 1992.

[10] Bony, J.-M., Principe du maximum dans les espaces de Sobolev, C. R. Acad. Sc. Paris 265, 333-336 (1967).

[11] Garnett, J. B., Bounded analytic functions, Academic Press, New York London Toronto Sydney San Francisco, 1981.

[12] Torchinsky, A., Real-variable methods in harmonic analysis, Academic Press, Orlando San Diego New York London Tokyo, 1986.

[13] John, F., Nirenberg, L., On functions of bounded mean oscillation, Comm. Pure and Appl. Math. 14, 415-426 (1961).

[14] Sarason, D., Functions of vanishing mean oscillation, Trans. Amer. Math. Soc. 207, 391-405 (1975).

[15] Bony, J.-M., Courrège, P., Priouret, P., Semi-groupes de Feller sur une variété à bord compacte et problèmes aux limites intégro-différentiels du second ordre donnant lieu au principe du maximum, Ann. Inst. Fourier (Grenoble) 18, 369-521 (1968).

[16] Yosida, K., Functional analysis, sixth edition, Springer-Verlag, Berlin Heidelberg New York, 1980.

[17] Adams, R. A., Sobolev spaces, Academic Press, New York, 1975.

[18] Bergh, J. and Löfström, J., Interpolation spaces, an introduction, SpringerVerlag, Berlin Heidelberg New York, 1976.

[19] Triebel, H., Theory of function spaces, Birkhäuser, Basel Boston Stuttgart, 1983.

[20] Taira, K., Logistic Dirichlet problems with discontinuous coefficients, J. Math. Pures Appl. 82, 1137-1190 (2003). 


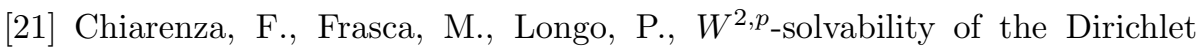
problem for nondivergence elliptic equations with VMO coefficients, Trans. Amer. Math. Soc. 336, 841-853 (1993).

[22] Chiarenza, F., Frasca, M., Longo, P., Interior $W^{2, p}$ estimates for nondivergence elliptic equations with discontinuous coefficients, Ricerche di Matematica 60, 149-168 (1991).

[23] Maugeri, A., Palagachev, D. K. and Softova, L. G., Elliptic and parabolic equations with discontinuous coefficients, Mathematical Research vol. 109, Wiley-VCH, 2000.

[24] Gilbarg, G. and Trudinger, N. S., Elliptic partial differential equations of second order, 1998 edition, Springer-Verlag, New York Berlin Heidelberg Tokyo, 1998.

[25] Troianiello, G. M., Elliptic differential equations and obstacle problems, Plenum Press, New York, 1987.

[26] Taira, K., Boundary value problems for elliptic integro-differential operators, Math. Z. 222, 305-327 (1996). 